\title{
Dinâmica espacial dos parâmetros físicos e químicos da água em viveiros de piscicultura
}

\section{Spatial dynamics of the physical and chemical parameters of water in fish farms}

\author{
José Mário Lopes da Rocha ${ }^{l}$; Antonio Clementino dos Santos ${ }^{2 *}$; Danilo Gomes de Oliveira ${ }^{l}$; Otacilio Silveira Júnior ${ }^{3}$; Elisson \\ Gomes da Silva ${ }^{4}$; Caio Leonardo Silva Ferreira ${ }^{4}$
}

\begin{abstract}
Resumo: O Brasil apresenta características que podem fazer do seu território o grande celeiro mundial para produção de organismos piscícolas cultivados. No entanto, para isso, é fundamental estudar os parâmetros físico e químico da água das áreas de cultivo, para que se possam buscar estratégias para evitar erros de manejos. Com isso, objetivou-se avaliar a dinâmica espacial dos parâmetros físicos e químicos da água de viveiros de piscicultura e sua relação de dependência. $O$ estudo foi realizado em um viveiro escavado em terreno natural, localizado em uma propriedade, adjacente à fazenda experimental da escola de Medicina Veterinária e Zootecnia, da Universidade Federal do Tocantins, no município de Araguaína. Os pontos para coleta no grid foram obtidos através de GPS, considerando as dimensões da área total. Em cada ponto georreferenciado, foram realizadas coletas em duas profundidades diferentes, uma a $20 \mathrm{~cm}$ da superfície da água (profundidade 1), e outra a 20 $\mathrm{cm}$ do fundo do tanque (profundidade 2), totalizando 108 amostras. Ocorreu grande variabilidade entre as profundidades para maioria das variáveis. Na estatística descritiva foi observado coeficiente de variação de grau moderado apenas para oxigênio dissolvido. $\mathrm{Na}$ análise dos semivariogramas, apenas a variável condutividade elétrica demonstrou efeito pepita puro. Foi verificada dependência espacial entre as variáveis estudadas nas duas profundidades. Através da geoestatística, foi possível evidenciar alteração na distribuição espacial dos parâmetros físicos e químicos: oxigênio dissolvido, temperatura da água e pH, mas não foi útil para condutividade elétrica, dada sua baixa variabilidade espacial. Os parâmetros físicos e químicos da água dos tanques de piscicultura são influenciados pelas práticas de manejo, sendo facilmente detectado através dos mapas de isolinhas.
\end{abstract}

Palavras-chave: Piscicultura; Krigagem; Variabilidade.

Abstract: Brazil presents, characteristics that can make of its territory, the world's great barn for the production of cultivated fish organisms. However, for this it is fundamental to study the physical and chemical parameters of the water in the growing areas, so that strategies can be sought to avoid handling errors. The aim of this study was to evaluate the spatial dynamics of the physical and chemical parameters of fish pond water and their relationship of dependence. The study was carried out in a nursery excavated on natural land, located on a property, adjacent to the experimental farm of the Veterinary Medicine and Animal Science School, Federal University of Tocantins, in the municipality of Araguaína. The points for collection on the grid were obtained by GPS, considering the size of the total area. In each georeferenced point samplings were performed in two different depths, one $20 \mathrm{~cm}$ from the tank bottom (depth first) and the other at $20 \mathrm{~cm}$ from the water surface (depth 2) totaling 108 samples. There was major variation between depths for most variables. Descriptive statistics was observed moderate variation coefficient only for dissolved oxygen. In the analysis of semivariograms, only the variable electrical conductivity showed pure nugget effect. spatial dependence was found between the variables at both depths. The mesh used was effective in showing the spatial distribution of physical - chemical parameters: dissolved oxygen, water temperature and $\mathrm{pH}$, but it was not useful for electrical conductivity, given its low spatial variability. The physical and chemical parameters of the water of the fish ponds are influenced by the management practices, being easily detected through the isoline maps.

Key words: Fish; Kriging; Variability.

\footnotetext{
*Autor para correspondência

Recebido para publicação em 31/03/2017; aprovado em 04/06/2017

${ }^{1}$ Docentes do Instituto Federal de Educação, Ciência e Tecnologia do Tocantins, campus Colinas do Tocantins-IFTO. e-mail: jose.lopes@ifto.edu.br; danilo.oliveira@ifto.edu.br.

${ }^{2 *}$ Professor Adjunto da Universidade Federal do Tocantins, Campus de Araguaína, e-mail: clementino@mail.uft.edu.br;

${ }^{3}$ Doutor e ${ }^{4}$ Mestre em Ciência Animal Tropical, da Universidade Federal do Tocantins, Campus de Araguaína, e-mail: otaciliosilveira@hotmail.com; elisson.gomes@uol.com.br,caio.leonardo@hotmail.com
} 


\section{INTRODUÇÃO}

O Brasil apresenta (água em abundância, grande número de espécies adaptadas, temperatura e condições climáticas) características primordiais para alavancar a produção de organismos piscícolas, com possibilidade de se tornar um dos principais produtores mundiais (SIDONIO et al., 2012).

$\mathrm{Na}$ produção de organismos cultivados em viveiros escavados, o monitoramento das propriedades físicas e químicas da água, ditam o sucesso do empreendimento. Parâmetros como temperatura, oxigênio dissolvido, $\mathrm{pH}$ e condutividade elétrica, somados à turbidez, alcalinidade, dureza e compostos nitrogenados, assumem papel central no desenvolvimento dos organismos aquáticos, devendo ser monitorados para a otimização da produção (OLIVEIRA, 2009).

No entanto, um problema comum verificado na maioria das fazendas piscícolas, no manejo produtivo, é a consideração de valores absolutos no monitoramento físico e químico da água. Fato este que acaba por refletir em conflitos e medidas de manejo inadequadas, haja vista o tanque de piscicultura funciona como ecossistema biológico complexo, repleto de variações em sua extensão e nas diferentes profundidades. Quando se tem variação dos atributos estudados, algumas técnicas estatísticas ajudam a predizer o comportamento espacial das variáveis, como é o caso da geoestatística, que permite estudar as variações espaciais que possam ocorrer, com base na dependência espacial dos atributos (VIEIRA, 2000).

Conhecer a variabilidade espacial dos atributos que interferem na qualidade da água permite práticas de manejo localizadas, que possam corrigir e entender as variações com práticas específicas para aplicação em diferentes atividades, dentre elas, a produção de peixes em tanques escavados (PLANT, 2001; SILVA; FERRARI, 2011).

Algumas técnicas estatísticas podem ser utilizadas como ferramentas de predição do comportamento espacial das variáveis, a exemplo da geoestatística, que permite estudar as variações espaciais que possam ocorre, com base na dependência especial dos atributos (VIEIRA, 2000). Diante do exposto, objetivou-se avaliar a dinâmica espacial dos parâmetros físicos e químicos da água e sua relação de dependência em viveiro de piscicultura.

\section{MATERIAL E MÉTODOS}

O estudo foi realizado em uma propriedade adjacente à fazenda experimental da escola de Medicina Veterinária e Zootecnia, da Universidade Federal do Tocantins, no município de Araguaína (TO), em viveiro escavado em terreno natural.

A região pertence ao bioma Amazônico, cujas coordenadas geográficas são $7^{\circ} 06^{\prime} 45^{\prime}$ S e $48^{\circ} 11^{\prime} 42^{\prime}$ 'W, com altitude média de $235 \mathrm{~m}$. O clima da região é o AW-Tropical quente e úmido, com verão úmido e período de estiagem no inverno, estando sob domínio climático tropical semiúmido, com estiagem de aproximadamente 4 meses (junho a setembro) e precipitação pluviométrica média anual de 1.800 $\mathrm{mm}$.

$\mathrm{O}$ viveiro utilizado apresentava formato regular, com dimensões de $85 \times 24 \mathrm{~m}$ e área total de $2.040 \mathrm{~m}^{2}$. A entrada de água do sistema, localiza-se na parte mais rasa e drenagem na parte mais profunda. Para realização da coleta, foi delineada uma malha regular ou grid de 5 x $6 \mathrm{~m}$ abrangendo a área total.

Os pontos para coleta no grid foram obtidos através de GPS (Garmin GPSMap 76CSx), considerando as dimensões da área total. Em cada ponto georreferenciado, foram realizadas coletas em duas profundidades diferentes, uma a $20 \mathrm{~cm}$ da superfície da água (profundidade 1), e outra a $20 \mathrm{~cm}$ do fundo do tanque (profundidade 2), totalizando 108 amostras. As coletas foram realizadas no período da manhã, das 7 às 11 horas, sendo aferidos os seguintes parâmetros: temperatura da água $\left({ }^{\circ} \mathrm{C}\right)$, oxigênio dissolvido da água (mg.L $\left.{ }^{1}\right)$ e condutividade elétrica $\left(\mu \mathrm{S} . \mathrm{cm}^{-1}\right)$, por meio de um oxímetro microprocessador modelo AT - 150, além do potencial hidrogeniônico $(\mathrm{pH})$, que foi analisado por meio de um pHmetro digital.

Os dados foram avaliados usando as estatísticas descritivas e geoestatística. Nas análises descritivas, foram calculadas média, mediana, coeficiente de variação $(\mathrm{CV})$, coeficiente de assimetria e curtose com o auxílio do software GS+ versão 7.0 (ROBERTSON, 1998). De acordo com os valores de $\mathrm{CV}$, as variabilidades dessas variáveis foram classificadas, segundo Warrick e Nielsen (1980), em baixa $(\mathrm{CV}<12 \%)$, média $(12 \%<\mathrm{CV}<62 \%)$ e alta $(\mathrm{CV}>62 \%)$.

As variabilidades espaciais foram determinadas por meio de exames de semivariogramas obtidos, ajustados a modelos teóricos para obtenção de seus parâmetros (pepita, patamar e alcance). Após o ajuste dos semivariogramas, os dados foram aplicados diretamente na interpolação por krigagem ordinária para a geração dos mapas das variáveis oxigênio dissolvido da água $\left(\mathrm{mg} . \mathrm{L}^{-1}\right)$, temperatura da água $\left({ }^{\circ} \mathrm{C}\right)$, condutividade elétrica $\left(\mu \mathrm{S} . \mathrm{cm}^{-1}\right)$ e potencial hidrogeniônico $(\mathrm{pH})$.

Para análise do grau de dependência espacial (GDE) das variáveis foi utilizada a relação $\mathrm{C} /(\mathrm{C}+\mathrm{C} 0)$, classificada, segundo Dalchiavon et al., (2012) como forte (GDE $\leq 0,25)$, moderada $(0,25 \leq \mathrm{GDE}<0,75)$ e baixa dependência $(\mathrm{GDE} \geq$ 0,75 ). Os modelos de semivariogramas considerados foram o linear, o esférico, o exponencial e o gaussiano, sendo ajustados por meio do programa GS+ versão 7.0. Após a escolha do melhor modelo de ajuste aos dados foi possível realizar a construção dos mapas de isolinhas pelo software GS+ versão 7.0, que realiza as interpolações utilizando-se o método de krigagem originária.

\section{RESULTADOS E DISCUSSÃO}

Não houve simetria nas variáveis estudadas. Através dos dados, verificam-se que as variáveis oxigênio dissolvido, condutividade elétrica e $\mathrm{pH}$ (profundidade 2) e oxigênio dissolvido e condutividade elétrica (profundidade 1), apresentaram assimetria positivas. Os dados que apresentaram assimetrias negativas foram temperatura da água e $\mathrm{pH}$ (profundidade 2) e temperatura da água (profundidade 1), respectivamente (Tabela 1). Os valores referentes à média e mediana, se mostraram próximos em todas as variáveis estudadas, demonstrando que os dados possuem ou se aproximam da distribuição normal (LITTLE; HILLS, 1978).

Os coeficientes de variação foram considerados baixos para temperatura da água, condutividade elétrica e $\mathrm{pH}$, nas duas profundidades estudadas, somente a variável oxigênio dissolvido apresentou coeficiente de variação médio (Tabela 1). 
Tabela 1. Resultado para as variáveis oxigênio dissolvido (O.D) (mg. $\left.\mathrm{L}^{-1}\right)$, temperatura da água $\left({ }^{\circ} \mathrm{C}\right)$, condutividade elétrica $\left(\mu \mathrm{S} . \mathrm{cm}^{-1}\right)$ e $\mathrm{pH}$, em viveiro escavado de terra em duas profundidades diferentes. Araguaína, Tocantins

\begin{tabular}{lcccccc}
\hline & Variáveis & Média & Med & CV & Assimetria & Curtose \\
\hline \multirow{4}{*}{ Profundidade 1 } & O.D $\left(\mathrm{mg} . \mathrm{L}^{-1}\right)$ & 15,05 & 14,3 & 29,05 & 0,27 & $-0,36$ \\
& Temp. água $\left({ }^{\circ} \mathrm{C}\right)$ & 29,22 & 29,3 & 0,23 & $-0,10$ & $-1,38$ \\
& Cond. Elétrica $\left(\mu \mathrm{S} . \mathrm{cm}^{-1}\right)$ & 0,03 & 0,03 & 1,7 & 0,09 & 7,1 \\
& $\mathrm{pH}$ & 5,93 & 5,99 & 0,32 & $-3,84$ & 18,75 \\
\hline Profundidade 2 & O.D $\left(\mathrm{mg} . \mathrm{L}^{-1}\right)$ & 5,37 & 4,4 & 14,4 & 0,57 & $-0,84$ \\
& Temp. água $\left({ }^{\circ} \mathrm{C}\right)$ & 29,62 & 29,7 & 0,49 & $-0,09$ & 0,32 \\
& Cond. Elétrica $\left(\mu \mathrm{S} . \mathrm{cm}^{-1}\right)$ & 0,03 & 0,03 & 1,77 & 1,17 & 0,53 \\
\hline
\end{tabular}

Profundidade 1 (20 cm da superfície da água); Profundidade 2 (20 cm do fundo do tanque). O.D: Oxigênio dissolvido; Med: Mediana; CV: Coeficiente de Variação

Segundo Silva et al. (2013), coeficientes de variação (CV) elevados são um dos principais indicadores da existência de heterogeneidade. Uma variação maior entre as profundidades foi observada no oxigênio dissolvido, com valores médios de 5,37 (mg. $\left.\mathrm{L}^{-1}\right)$, para profundidade 2 e 15,05 $\left(\mathrm{mg} . \mathrm{L}^{-1}\right)$, para profundidade 1 , respectivamente. Segundo Bambi et al. (2008), em lagos e reservatórios, como represas e tanques escavados, devido à estratificação térmica e outros fatores, como carga orgânica elevada, a concentração de oxigênio dissolvido varia com a profundidade, sendo maior na superfície e menor no fundo.

Para os semivariogramas, os modelos obtidos nas diferentes variáveis mensuradas mostram na profundidade 2 , alto grau de dependência espacial para as variáveis: oxigênio dissolvido, temperatura da água e $\mathrm{pH}$. Na profundidade 1 , verificou-se alto grau de dependência espacial para temperatura da água e $\mathrm{pH}$, e grau moderado para oxigênio dissolvido. Essa maior dependência espacial é esperada uma vez que, nos viveiros escavados, sua construção é seguida com alguns padrões como: entrada de água em uma das extremidades e saída na outra extremidade, com declividade próxima de $5 \%$. No local de entrada fonte de água dos viveiros é esperado que a água apresenta-se mais limpa, com condutividade baixa e à medida que essa água vai se deslocando e recebendo sedimentos e dejetos vão reduzindo sua qualidade até chegar a saída. Tornando, assim, os parâmetros físicos e químicos heterogêneo em toda área do tanque. No entanto, pode-se amenizar os problemas ocasionados pelas práticas de manejos.

A variável condutividade elétrica, apresentou efeito pepita puro em ambas situações, sendo então considerados valores médios (Tabela 2). $\mathrm{Na}$ profundidade 2, os semivariogramas de oxigênio dissolvido, temperatura da água e $\mathrm{pH}$, se ajustaram ao modelo gaussiano, sendo que somente, condutividade elétrica se ajustou ao modelo esférico.

Tabela 2. Modelos e parâmetros estimados dos semivariogramas experimentais para as variáveis oxigênio dissolvido (O.D) $\left(\mathrm{mg} . \mathrm{L}^{-1}\right)$, temperatura da água $\left({ }^{\circ} \mathrm{C}\right)$, condutividade elétrica $\left(\mu \mathrm{S} . \mathrm{cm}^{-1}\right)$ e $\mathrm{pH}$, em viveiro escavado de terra em duas profundidades diferentes. Araguaína, Tocantins

\begin{tabular}{|c|c|c|c|c|c|c|c|c|}
\hline & Variáveis & Modelo & $(\mathrm{C} 0)$ & $(\mathrm{C} 0+\mathrm{C} 1)$ & $\mathrm{A}$ & & $\overline{\mathrm{DE}}$ & $\mathrm{R}^{2}$ \\
\hline \multirow{4}{*}{ Profundidade 1} & O.D (mg.L L $\left.^{-1}\right)$ & exponencial & 9,14 & 28,27 & 6,01 & 32,33 & Moderado & 0,71 \\
\hline & T. água $\left({ }^{\circ} \mathrm{C}\right)$ & gaussiano & 0,04 & 0,49 & 29,16 & 8,16 & Alto & 0,97 \\
\hline & C.E. $\left(\mu\right.$ S.cm $\left.{ }^{-1}\right)$ & EPP & - & - & - & - & - & 0,26 \\
\hline & $\mathrm{pH}$ & exponencial & 0,01 & 0,32 & 2,62 & 3,12 & Alto & 0,12 \\
\hline \multirow{4}{*}{ Profundidade 2} & O.D (mg.L L $\left.^{-1}\right)$ & gaussiano & 3,57 & 26,8 & 23,87 & 13,32 & Alto & 0,97 \\
\hline & T. água $\left({ }^{\circ} \mathrm{C}\right)$ & gaussiano & 0,14 & 0,89 & 24,23 & 15,73 & Alto & 0,97 \\
\hline & C.E. $\left(\mu\right.$ S.cm $\left.{ }^{-1}\right)$ & EPP & 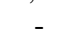 & - & - & - & - & 0,82 \\
\hline & $\mathrm{pH}$ & gaussiano & 0,05 & 0,28 & 21,12 & 17,86 & Alto & 0,96 \\
\hline
\end{tabular}

*C0: Efeito Pepita; $(\mathrm{C} 0+\mathrm{C} 1)$ : Patamar; A: Alcance; GDE: grau de dependência espacial (\%); EPP: Efeito pepita puro; O.D: oxigênio dissolvido; T. água: temperatura da água; C.E. Condutividade Elétrica; Profundidade 1 (20 cm da superfície da água); Profundidade 2 (20 cm do fundo do tanque).

Na profundidade 1, oxigênio dissolvido e $\mathrm{pH}$, se ajustaram ao modelo exponencial, temperatura da água, ao modelo gaussiano e condutividade elétrica ao modelo esférico (Tabela 2). Segundo Miles et al. (2008), quando os semivariogramas se ajustam a algum dos modelos observados, existe um ponto a partir de um determinado valor da distância entre pontos amostrados, na qual não mais se observa dependência espacial, isto é dado pelo fato desses modelos possuírem patamares. Os coeficientes de determinação $\left(\mathrm{r}^{2}\right)$ indicam a qualidade do ajuste do modelo de semivariograma teórico experimental, sendo que valores próximos da unidade indicam bom ajuste (SILVA; FERRARI, 2011).

De acordo com o mapa de isolinhas, verifica-se grande amplitude para oxigênio dissolvido, em toda extensão do viveiro nas duas profundidades. $\mathrm{Na}$ profundidade 2 , os valores mínimos e máximos observados foram de $0,6 \mathrm{mg} . \mathrm{L}^{-1}$ e 12,6 mg. $\mathrm{L}^{-1}$, respectivamente (Figura $1 \mathrm{~A}$ ).

Os valores mínimos desse parâmetro, foram mais enunciados na parte final do tanque, ou seja, no local de saída de água. Isso se dá em função do fluxo de água que segue esta direção, carregando consigo, dentro de toda extensão do tanque, partículas orgânicas advindas da adubação e restos de alimentos, fatores que reduzem em sobremaneira a quantidade de oxigênio. Segundo Silva e Ferrari (2012), o oxigênio dissolvido é um dos parâmetros físicos e químicos mais importantes para a sobrevivência dos organismos cultivados. Quando os níveis nos tanques tornam-se baixos, menores que 5 mg.L.-1 (LIMBERGER; CORRÊA, 2005), para maioria das espécies, pode acarretar estresse fisiológico, enfraquecimento do sistema imunológico, doenças e até a morte. 
Os maiores valores de oxigênio dissolvido (12,6 mg.L ${ }^{1}$ ), na profundidade 2 , foram observados no centro do viveiro, local onde manejos como calagem, e, principalmente, adubação orgânica, foram intensificados durante o ciclo de produção, fatores que influenciaram na evolução da população planctônica nesse ponto, refletindo assim no aumento das taxas de oxigênio dissolvido (Figura 1A).

Figura 1. Distribuição espacial das variáveis oxigênio dissolvido $\left(\mathrm{mg} \cdot \mathrm{L}^{-1}\right)$, $(\mathrm{A}$ e $\mathrm{B})$ temperatura $\left({ }^{\circ} \mathrm{C}\right)(\mathrm{C}$ e D), condutividade elétrica $\left(\mu \mathrm{S} . \mathrm{cm}^{-1}\right)(\mathrm{E} \mathrm{e} \mathrm{F})$ e pH da água $(\mathrm{G} \mathrm{e} \mathrm{H})$ em duas profundidades, em viveiro de terra escavado, Araguaína, Tocantins
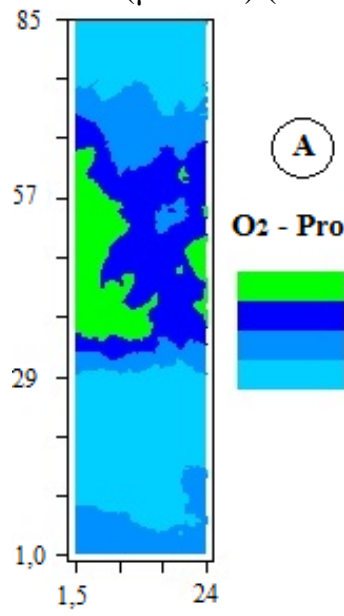

O2 - Prof. 02
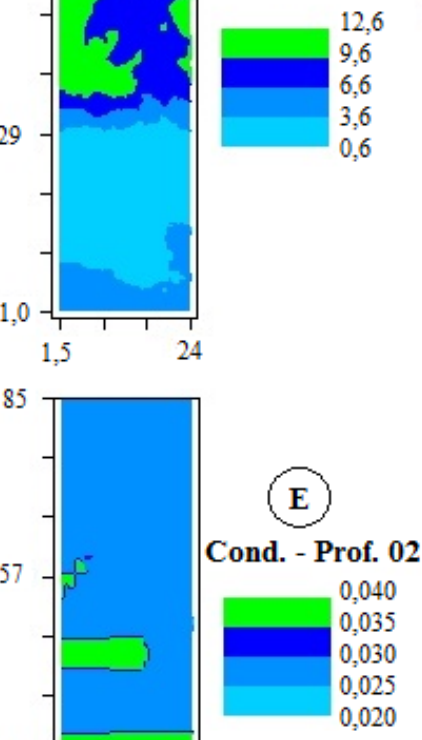
concentração de nutrientes nas águas de tanques de piscicultura depende das práticas de manejo e da coluna d'água que entra no sistema. Algumas práticas podem elevar a concentração de nutrientes, como é o caso do uso de fertilizantes orgânicos e inorgânicos, que irá refletir em aumento da concentração de fitoplâncton, que através do processo de fotossíntese, se torna a principal fonte de oxigênio. Outro fator que explica essa maior concentração de oxigênio nesse ponto, é a temperatura, que se mostrou reduzida nessa região do viveiro, e segundo Araújo et al. (2015), normalmente, temperaturas menores elevam a concentração de oxigênio dissolvido na água.

$\mathrm{Na}$ profundidade 1 , os menores e maiores valores foram, respectivamente, 5,6 e 24,3 mg.L-1 (Figura 1B). O fato da mensuração ter sido realizada próxima à superfície da água, contribuiu bastante para elevação do oxigênio, fato que ocorre em função de trocas gasosas entre atmosfera e a água do viveiro, mediante processo de difusão (MALLASEN, et al., 2012).

A temperatura da água, variou de 28,3 a $30,6{ }^{\circ} \mathrm{C}$, na profundidade 2 (Figura 1C) e de 28,5 a $29,8{ }^{\circ} \mathrm{C}$, na profundidade 1 (Figura 1D). Observa-se que em ambas profundidades, as menores temperaturas foram verificadas
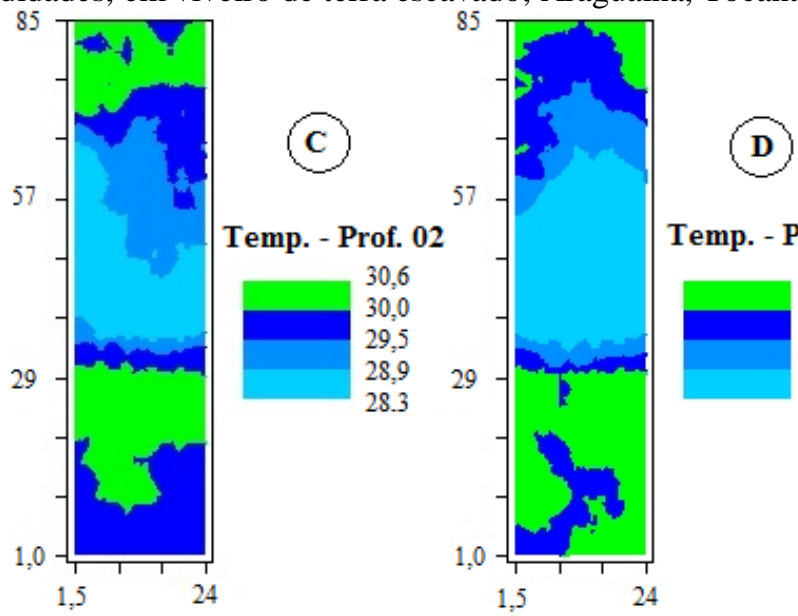

Temp. - Prof. 01
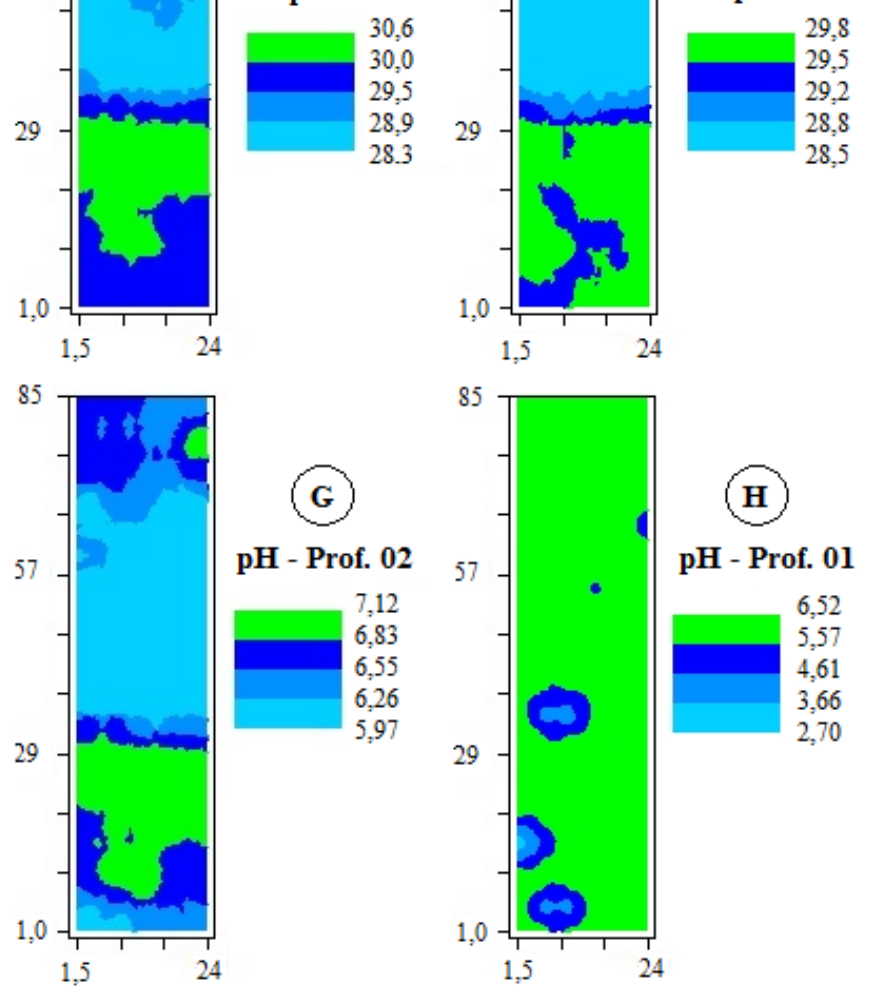

justamente nos locais onde ocorreram as práticas de manejo, com a adubação orgânica contribuiu para elevar o crescimento de fitoplâncton e zooplâncton, o que favoreceu também para o aumenta da turbidez da água, contribuindo para redução da incidência de luz que chega nas camadas mais profundas do viveiro, como pode ser observado com a redução da temperatura nesse local. De acordo com Xie et al. (2011), um dos principais fatores que afetam as respostas fisiológicas dos peixes é a temperatura da água, isso irá refletir no crescimento e no consumo de ração, existindo relação direta entre aumento da temperatura e consumo de alimento (CERQUEIRA, 2010).

Para condutividade elétrica, não foram verificadas grandes variações entre as profundidades e na extensão do viveiro, sendo observados os maiores valores $\left(0,04 \mu \mathrm{S} . \mathrm{cm}^{-1}\right)$ em uma região isolada, próxima à entrada de água (Figura $1 \mathrm{E}$ e F). Para Esteves (1988), a condutividade elétrica constitui uma das variáveis mais importantes em limnologia, visto poder fornecer importantes informações, por exemplo, a respeito de principais atividades de um ecossistema aquático, como produção (redução dos valores) e decomposição (aumento dos valores).

$\mathrm{O}$ mapa de isolinhas referente $\mathrm{pH}$ mostra o quanto essa característica é responsiva às práticas de manejo 
realizadas no viveiro. Para a profundidade 2 , naquele local onde foi concentrada a fertilização com adubos orgânicos, foram observados os menores valores de $\mathrm{pH}$ (Figura 1G). Segundo Bambi et al. (2008), as flutuações dos pHs ácidos, neutro e/ou alcalino estão relacionadas com os processos de decomposição e as atividades fotossintéticas. A acidez do $\mathrm{pH}$ na água é comum quando a atividade predominante é a decomposição da matéria orgânica, que resulta da atividade do fitoplâncton e do material autóctone proveniente de detritos. Na profundidade 1, a variabilidade foi baixa, ocorrendo pouca variação no decorrer do viveiro, haja vista as mensurações terem sido realizadas próximas à superfície da água, local de pequena circulação de resíduos orgânicos, baixa liberação de íons hidrogênio, e consequentemente baixa variação nos valores de $\mathrm{pH}$ (Figura 1H).

Pode-se verificar que os tanques de piscicultura apresentam dependência espacial entre as variáveis estudadas nas duas profundidades.

\section{CONCLUSÕES}

Os parâmetros físicos e químicos da água dos tanques de piscicultura são influenciados pelas práticas de manejo.

Nas práticas de manejos adotadas nos tanques deve ser levada em conta toda extensão da área, para evitar zonas com qualidade da água inferior aos adequados para a criação dos peixes.

\section{AGRADECIMENTOS}

Ao CNPq e Capes pelo suporte financeiro e bolsas (Mestrado e Produtividade em Pesquisa) para condução da pesquisa.

\section{REFERÊNCIAS}

ARAUJO, D. M.; FERNANDES JUNIOR, A. C.; TEIXEIRA, C. P.; PEZZATO, L. E.; BARROS M. M. Perfil hematológico de tilápias-do-nilo alimentadas com dietas contendo diferentes lipídeos e estimuladas por baixa temperatura. Revista Caatinga, Mossoró, v. 28, n. 1, p. $220-$ 227, 2015.

BAMBI, P.; DIAS, C. A. A.; SILVA, V. P. Produção Primária do Fitoplâncton e as suas relações com as principais variáveis limnológicas na Baía das Pedras, Pirizal Nossa senhora do Livramento, Pantanal de Poconé - MT. Revista Uniciências, v.12, 2008, p. $47-64$.

CERQUEIRA, V. R. Cultivo de robalopeva (Centropomus parallelus). In: BALDISSEROTTO, B.; GOMES, L. de C. (Ed.). Espécies nativas para piscicultura no Brasil. 2.ed. Santa Maria: UFSM, p.489-520, 2010.

DALCHIAVON, F. C.; CARVALHO, M. P.; ANDREOTTI, M.; MONTANARI, R. Variabilidade espacial de atributos da fertilidade de um Latossolo Vermelho Distroférrico sob Sistema Plantio Direto. Revista Ciência Agronômica. Fortaleza, v. 43, n. 3, 2012.

ESTEVES, F. A. Fundamentos de limnologia. Rio de Janeiro: Interciências/ FINP, 602p. 1988.

LITTLE, T. M.; HILLS, F. J. Agricultural experimentation. New York: J. Wiley \& Sons, 1978. 350p.

LIMBERGER, L.; CORRÊA, G. T. Diagnóstico ambiental do ribeirão Lindóia (Londrina-PR). Aspectos físico-químicos e bacteriológicos. Revista Eletrônica da Associação de Geógrafos Brasileiros, v.2, n.2, p.16-23, 2005.

MACEDO, C. F.; SIPAÚBA-TAVARES, L. H. Eutrofização e qualidade da água na piscicultura: consequências e recomendações. Boletim do Instituto de Pesca, São Paulo, v.36, n.2, p.149 - 163, 2010.

MALLASEN, M.; CARMO, C. F.; TUCCI, A.; BARROS, H. P.; ROJAS, N. E. T.; FONSECA, F. S.; YAMASHITA, E. Y.; Qualidade da água em sistema de piscicultura em tanquesrede no reservatório de ilha solteira, sp. Boletim do Instituto de Pesca, São Paulo, v.38, n.1, p.15 - 30, 2012.

MILES, D. M.; ROWE, D. E.; OWENS, P. R. Winter broiler litter gases and nitrogen compounds: temporal and spatial trends. Atmospheric Environment, v.42, p.3351-3363, 2008.

OLIVEIRA, R. Nota Técnica: Panorama Geral da Aquicultura no Brasil. Associação Para a Produção Sustentável (APS). Ituberá - Bahia - Brasil. Ed. 1, outubro de 2009.

PLANT, R. E. Site specifies management: the application of production of information technology to crop production. Computers and Electronics in Agriculture, Amsterdam, v. 30, n. 1, p. 9 - 29, 2001.

ROBERTSON, G. P. Geostatistics for the environmental sciences - GS+ User's guide. Plainwell: Gamma Design Soffware, 1998. 152p.

SIDONIO, L.; CAVALCANTI, I.; CAPANEMA, L.; MORCH, R.; MAGALHÃES, G.; LIMA, J.; BURNS, V.; ALVES JÚNIOR, A. J.; MUNGIOLI, R. Panorama da aquicultura no Brasil: desafios e oportunidades. BNDES Setorial 35, p. $421-463,2012$.

SILVA, S. F; FERRARI, J. L. Variabilidade espacial de parâmetros físico-químicos da água em viveiros de piscicultura. In: II Simpósio de Geoestatística em Ciências Agrárias, 2011, Botucatu. Anais... Botucatu: UNESP, 2011, p. $35-41$.

SILVA, S. F; FERRARI, J. L. Análise espacial de atributos físico químicos da água em viveiros de piscicultura com geometrias diferentes. Enciclopédia Biosfera, v.8, N.14; p. 5 $1,2012$.

SILVA, E. G; SANTOS, A. C; FERREIRA, C. L. S; SOUSA, J. P. L; ROCHA, J. M. L; SILVEIRA, O. J. Variabilidade espacial das características ambientais e peso de frangos de corte em galpão de ventilação negativa. Revista Brasileira de Saúde Produção Animal, v.14, n.1, p.132-141 2013.

VIEIRA, S. R. Geoestatística em estudos de variabilidade do solo. In: NOVAIS, P. F.; ALVAREZ, V. H.; SCHAEFER, C. E. G. R. (Eds.) Tópicos em ciência do solo. Sociedade Brasileira de Ciência do Solo. v. 1, p.1- 54, 2000.

WARRICK, A. W.; NIELSEN, D. R. Spatial variability of soil physical properties in the field. In: HILLEL, D. (Ed.). Applications of soil physics. New York: Academic, p.319344, 1980.

XIE, S.; ZHENG, K.; CHEN, J.; ZHANG, Z.; ZHU, X.; YANG, Y. Effect of water temperature on energy budget of Nile tilapia, Oreochromis niloticus. Aquaculture Nutrition, v.17, p.683-690, 2011. 